\title{
Desarrollo organizacional y los procesos de cambio en las instituciones educativas, un reto de la gestión de la educación
}

\section{Organizational Development and Change Processes in Educational Institutions, a Challenge for The Management of Education}

\author{
Guiselle M. Garbanzo-Vargas ${ }^{1}$ \\ Universidad de Costa Rica \\ Escuela de Administración Educativa \\ San José, Costa Rica \\ gmgarban@gmail.com
}

Recibido: 29 agosto 2014 Aceptado: 25 junio 2015 Corregido: 19 julio 2015

\begin{abstract}
Resumen. Con el propósito de gestionar los distintos procesos por los que atraviesan las organizaciones educativas en aras de alcanzar su desarrollo organizativo, y responder así a las demandas emergentes del entorno, el presente artículo tiene como objetivo realizar una revisión conceptual de los principales fundamentos asociados al desarrollo organizacional. Se toman en cuenta la gestión de los procesos de cambio que deben ejecutarse y el papel de la administración de la educación desde la gestión que, mediante un liderazgo transformador, debe actuar desarrollando organizaciones inteligentes, con culturas renovadas, dispuestas a gestar los cambios necesarios para alcanzar la visión organizacional. Sobresale, entre sus consideraciones finales, que la gestión de la educación posee un rol fundamental en la conducción eficiente de las organizaciones educativas, desarrollándolas como organizaciones inteligentes, de manera que alcancen altos estándares de calidad en el servicio que ofrecen, traducido en una mejor formación según la población meta a la cual se dirige.
\end{abstract}

Palabras clave: Administración de la educación, gestión de la educación, gestión del conocimiento, organizaciones inteligentes, coordinación en la educación, desarrollo organizacional, administración universitaria y cambio organizacional.

1 Docente catedrática de la Universidad de Costa Rica, actualmente es la directora del Posgrado en Ciencias de la Educación con énfasis en Administración Educativa, directora de la Revista Científica Gestión de la Educación. Es investigadora en la Escuela de Administración Educativa de la Facultad de Educación de la Universidad de Costa Rica, coordinadora de la Sede Instituto Gestión de Liderazgo Universitario (IGLU), Región Centroamérica y es miembro de la Red de Mujeres Líderes de la Educación superior EMULIES de la Organización Interamericana Universitaria (OUI). Es Doctora en Educación de la Universidad de Costa Rica con pasantía de investigación en la Universidad de Salamanca, España, Magister en Administración Educativa de la Universidad de Costa Rica, Licenciada en Ciencias de la Educación con énfasis en Orientación de la Universidad de Costa Rica, Licenciada en Administración con énfasis en Recursos Humanos de la Universidad Nacional y Bachiller en Ciencias de la Educación con énfasis en Orientación de la Universidad de Costa Rica. Ha realizado pasantías académicas en la Universidad de Helsinki, Finlandia, Universidad de Murcia, España, Universidad de Montreal - Organización Universitaria Interamericana, también en la Unesco, Suiza, Ginebra y Universidad de Victoria, Canadá. También ha participado en distintos congresos Internacionales, entre ellos en Brazil, México, Argentina, España y China. Ha publicado distintos artículos en el campo de la educación superior pública y de la administración de la educación en revistas nacionales e internacionales, ha impartido varias conferencias en el ámbito nacional e internacional y ha dirigido el II, III y IV Congreso Internacional de Administración de la Educación y ha sido directora de la Escuela de Administración Educativa, Facultad de Educación de la Universidad de Costa Rica en dos períodos. También es evaluadora experta en distintas revistas científicas a nivel nacional e internacional y evaluadora del Sistema de Acreditación de la Educación Superior (SINAES), Costa Rica. 


\begin{abstract}
For the purpose of managing the different processes that educational organizations face in order to reach their organizational development and thus satisfy emergent claims of the context, the present article offers a conceptual review of the principal concepts associated with organizational development. It includes the management of the change processes required as well as the role of the administration of education which must develop intelligent organizations with improved cultures, willing to generate the necessary changes to achieve the organizational vision. One of its final considerations is that educational management has a key role in the efficient guidance of educational organizations, developing them as intelligent organizations so that they meet high standards in the service they provide which translates into better education for the target population.
\end{abstract}

Key Words: Educational Administration, Educational management, intelligent organizations, organizational development, University Administration, and organizational change.

\title{
Introducción
}

En los albores del presente siglo son muchos los desafíos por los que atraviesan las organizaciones en general para asegurar un lugar pertinente en la desafiante sociedad. De estas exigencias no se escapan las organizaciones educativas, las cuales, debido a su responsabilidad social en el proceso formativo del ser humano, deben enfrentarse a importantes y complejas disyuntivas con respecto a su actuar y sus procesos de organización con miras a un permanente desarrollo, que les permita ofrecer procesos educativos de alto nivel.

La dinámica de la actual sociedad ha dejado atrás paradigmas organizacionales tradicionales para darle lugar a nuevas concepciones organizacionales sustentadas en el desarrollo con un enfoque de organizaciones inteligentes. Organizaciones que están en constante aprendizaje consigo mismas y con su entorno, donde los ambientes de aprendizaje están en constante construcción desde una visión sistémica e innovadora, que además de transformarlas las desarrolle en forma acertada, según las demandas del convulso y exigente entorno social.

Ninguna organización puede permitirse quedarse atrás o perder viabilidad en el contexto social y permanecer sin trascender, mucho menos las enfocadas al servicio educativo. Estas requieren, para el sano desarrollo social, ser pioneras, líderes en su quehacer de manera que sus esfuerzos sean plasmados en forma articulada con las demandas sociales sin poner en segundo lugar el desarrollo pleno del individuo. En consecuencia, están llamadas a generar estrategias que les permitan el desarrollo organizacional, que actúen como organizaciones inteligentes desde su estructura y misión y visión.

Precisamente desde este contexto y con el propósito de ofrecer lineamientos estratégicos para gestionar los distintos procesos por los se enfrentan las organizaciones en su cotidianidad, se presenta en este artículo una revisión conceptual de los principales fundamentos asociados al desarrollo organizacional, así como las condiciones necesarias para que las organizaciones educativas actúen y se desarrollen como organizaciones inteligentes, que aprenden de sí mismas y de su entorno. Se toma en cuenta la gestión de los procesos de cambio que deben ejecutar y el papel de la gestión de liderazgo de estos procesos. Además, se desarrollan aquellos componentes fundamentales de las organizaciones inteligentes como poseedoras de culturas renovadas, donde el papel de la gestión posee un rol fundamental en la conducción eficiente de las organizaciones educativas, de manera que alcancen altos estándares de calidad en el 
servicio que ofrecen, traducido en una mejor formación del ser humano, como individuo pleno consigo mismo y con su entorno hacia el bien común.

\section{Las organizaciones, conceptualización}

Las tendencias mundiales asociadas a la globalización y la denominada sociedad de la información y el conocimiento evidencian exigencias de transformaciones y desafíos para las organizaciones con una connotación especial en las organizaciones educativas, por su naturaleza con respecto a su papel formativo permanente ante la sociedad. En este contexto, las organizaciones deben responder con solidez y pertinencia asegurando su viabilidad social.

Las organizaciones no son las que manifiestan determinados comportamientos, son sus integrantes en el plano individual y grupal: ellos participan, actúan en distintas modalidades según la naturaleza de la misma organización. Estas no funcionan al azar y el éxito que se alcanza es producto de un conjunto de sinergias que, conducidas en cierta dirección, lo logran. El éxito no viene en forma aleatoria, las organizaciones son células sociales que responden a estímulos del ambiente tanto interno como externo y, según estos, son sus productos, sus comportamientos.

Las organizaciones requieren de una conducción, una administración. Para iniciar el desarrollo organizacional, además de ubicar las características ambientales, es pertinente conocer el capital humano con que cuenta, sus talentos en función de los aportes que se esperan de cada una de las partes. A criterio de Chiavenato (2009), existen tres variables independientes que requieren ser comprendidas en las organizaciones para el logro de los objetivos planteados, las cuales son la base para el desarrollo organizacional: el sistema organizacional, el nivel grupal y el nivel individual.

El sistema organizacional: Se refiere a la totalidad de la organización, esta se mira y se comprende como un todo conformado por componentes como cultura organizacional, diseño, procesos de trabajo, normativas, entre otros. Se parte de que el todo es diferente a cada una de las partes y estas, en conjunto, asumen un comportamiento distinto según las interacciones y propósitos planteados.

Nivel grupal: Alude a conductas manifiestas a nivel grupal, mediante el trabajo en equipo, el cual es diferente al comportamiento individual. El grupo tiene una serie de actitudes que conforman una sinergia particular, que determinan el desempeño organizacional de manera positiva o negativa, esta fuerza es un elemento estratégico a considerar por el liderazgo, quienes además de conocerlas deben comprenderlas y saber conducirlas según los propósitos de la organización y en consecuencia con su misión y visión.

Nivel individual: Plantea las características personales de cada individuo tales como sus competencias, formación académica, historia, actitudes, valores, personalidad, percepción, entre otras. Desde el momento que las personas ingresan a una organización tienen un grado de influencia según estas características propias y, a su vez, la organización también influye sobre ellas. Es transcendental un conocimiento acertado sobre los potenciales de cada individuo y sus comportamientos ante la organización, de manera que la organización sepa conducir a sus integrantes con solidez y pertinencia, para así ofrecer respuestas organizacionales competentes, según el escenario social al cual se debe la organización. 
Las organizaciones deben desarrollar la capacidad de responder en forma acertada, diligente y pertinente a los desafíos del contexto emergente, de manera que sean capaces de reconocer las exigencias sociales y desarrollar procesos de cambio que conduzcan a la organización con efectividad y calidad en función de la realidad social, en procura de ofrecer mejores oportunidades tanto para la misma organización como para la sociedad. Desde esta perspectiva, es necesaria una gestión capaz de comprender cómo se desarrollan los procesos de cambio en las organizaciones.

\section{El cambio organizacional, consideraciones en su desarrollo}

El cambio organizacional es considerado por Hellriegel, Jackson y Solcum (2005) como cualquier transformación asociada al diseño o en el funcionamiento de una organización. El personal directivo eficiente y eficaz sabe el momento oportuno en que se requiere un cambio y también conoce las estrategias de conducir a su organización mediante el proceso del cambio. Los cambios pueden ser promovidos por fuerzas internas o externas. En lo externo, suele suceder como consecuencia de las transformaciones sociales producto de la dinámica y compleja sociedad, así como también al conocer de buenas prácticas aplicadas en otras organizaciones. En lo interno, por necesidades propias de la organización tales como los procesos normales de ajustes estructurales y cambios en sus objetivos.

A nivel interno, debido a la necesidad de adoptar nuevos procesos, objetivos y métodos, es probable que la organización requiera aplicar cambios organizacionales transcendentales. Un ejemplo de estos cambios necesarios ha sido la implementación de las tecnologías en las organizaciones, que ha venido a representar nuevos métodos y procesos en su funcionamiento. Los cambios en las organizaciones se pueden producir de forma radical o en forma pausada.

El cambio en las organizaciones puede ser definido en grados, como radical o progresivo y por la oportunidad, como reactivo o anticipatorio (Hellriegel et. al., 2005). Los cambios radicales se dan cuando las organizaciones producen innovaciones en sus métodos de hacer las cosas, es el repensar de la organización, por lo general son difíciles de manejar. El cambio "pausado" o progresivo es un proceso de evolución continuo en el tiempo, donde se dan diversos ajustes en menor grado a los procesos internos de la organización.

Lewin, citado por Hellriegel et al. (2005), se refiere a una serie de etapas del proceso de cambio radical:

- Descongelamiento: es la preparación por parte de la administración para que sus miembros asuman una transformación importante.

- Transición: es la puesta en práctica del cambio.

- Recongelamiento: la solidificación del cambio. En esta etapa se requiere de una revisión constante para que los miembros que implementan el cambio no recaigan en los antiguos hábitos.

Existen dos momentos para que se produzca el cambio: cuando las organizaciones experimentan un deterioro en el desempeño (reactivo) o en forma anticipada gracias a la 
visión del liderazgo. En este último caso, como no hay una crisis evidente, esos pueden ser debidamente planeados y ejecutados en forma gradual. La capacidad de responder en forma eficiente a los diferentes cambios a los que se enfrentan las organizaciones determina su sostenibilidad y posicionamiento social. En general, organizaciones que logran conservar altos niveles de posicionamiento y sostenibilidad, a lo interno poseen culturas de dinamismo sistémico hacia el entorno; y -a lo interno- tienen visión proactiva, no reactiva, poseyendo la capacidad de acertar acciones eficientes para implementar el cambio, así como conocer sobre el momento oportuno de actuar.

$\mathrm{Al} \mathrm{respecto,} \mathrm{las} \mathrm{organizaciones} \mathrm{pueden} \mathrm{aplicar} \mathrm{distintas} \mathrm{acciones} \mathrm{para} \mathrm{la} \mathrm{implementación}$ efectiva del cambio con el propósito de mejorarla:

Elaborar iniciativas en forma gradual en los métodos empleados con una constante revisión en la evolución de estos hasta lograr la acción proyectada, según el objetivo a alcanzar.

- Capacidad de influir en los demás, proyectando y obteniendo legitimidad que permita a sus miembros canalizar sus esfuerzos hacia la dirección definida, implica credibilidad de parte de quien conduce la organización hacia el cambio esperado.

- Desarrollar habilidades efectivas para obtener de sus integrantes el compromiso y responsabilidad requerida, implica capacidad para persuadir en busca de los objetivos proyectados.

- Actuar con en forma constante en la dirección proyectada es saber desviar los obstáculos que suelen presentarse en todo proceso de cambio y asumir una actitud persistente hasta alcanzar el objetivo.

El cambio también requiere planeación, de esta forma es más ordenado y permite que las personas se preparen y participen de la planeación mediante distintos procesos. En este caso, la planeación busca proporcionar, a miembros de la organización, de medios útiles para dirigirse en forma exitosa, a pesar de las exigencias que tanto la organización como la sociedad demandan; una sociedad cada vez más crítica e informada de la calidad de los servicios que recibe y que son indispensables para la permanencia. Los procesos a considerar para la planeación efectiva del cambio, según Hellriegel et al. (2005) son:

- Evaluar el medio ambiente

- Determinar la brecha del desempeño

- Diagnosticar problemas organizacionales

- Articular y comunicar una visión para el futuro

- Desarrollar y poner en práctica el plan acción

- Anticipar las resistencia y tomar acciones para reducirla

- Vigilar los cambios

Dada su relevancia para las organizaciones desde la planeación efectiva del cambio, seguidamente se hace referencia a la aplicación de estos procesos desde la gestión. 
Evaluar el medio ambiente: Las organizaciones se enfrentan en la actualidad a un ambiente externo acelerado de transformaciones que exigen cambios para garantizar su sostenibilidad. Se destacan entre las principales fuerzas que actúan: la población a quien se dirige el servicio, la tecnología, la competencia y la fuerza de trabajo. Todo lo anterior no descarta la globalización. Las organizaciones educativas deben desarrollar habilidades acertadas para leer el contexto y realizar los ajustes más convenientes. Las competencias profesionales de su personal, así como la gestión empleada son la clave, las organizaciones inteligentes consideran la inversión en el desarrollo profesional como un ingreso y no un costo.

Determinar la brecha del desempeño: Es el análisis en torno a lo que la organización hace en relación con lo que se desea alcanzar. Las organizaciones inteligentes saben reconocer esta diferencia y proponer acciones estratégicas.

Diagnosticar problemas organizacionales: Es la capacidad de determinar, en forma clara, los problemas organizacionales, sus causas y valorar soluciones que permitan desarrollar la organización del estado en que se encuentra.

Articular y comunicar una visión para el futuro: Es necesario desarrollar, en sus miembros, entusiasmo, compromiso y claridad de las acciones a implementar, para lo cual es un requisito que el liderazgo de la institución tenga una visión acertada del futuro y pueda conducir en forma eficiente el cambio requerido.

Desarrollar y poner en práctica el plan acción: Para la implementación de los cambios, las organizaciones requieren desarrollar planes institucionales que contengan las metas definidas y los criterios evaluativos que permitan conocer sus alcances. Es necesario tener alternativas para encausar los posibles desvíos del plan, se construye y se desarrolla con la participación de la totalidad de miembros de la organización, para lo que la capacidad de integrarles es un reto para el liderazgo. El monitoreo es continuo; no puede verse como una acción al final.

Anticipar la resistencia y tomar acciones para reducirla: La resistencia siempre será una variable a considerar y puede darse por diferentes razones, como temores, malestar e intereses particulares. El personal directivo eficaz conoce y comprende las posibles resistencias y asume un papel de alerta para enfrentarlas.

Vigilar los cambios: Se requiere un monitoreo constante de los resultados, así como de los comportamientos de sus miembros como el nivel de motivación, satisfacción e involucramiento. Las acciones de implementación del cambio no tienen un carácter estable, a como actúan las fuerzas externas e internas de la organización, estas también pueden sufrir modificaciones que atentan con la implementación del cambio requerido.

\section{Rediseño organizacional para implementar el cambio}

La implementación de los cambios es una tarea compleja; implica rediseñar estructuras, procesos, rutinas a lo interno de la organización. Estas modificaciones deben planearse adecuadamente y deben responder a las metas y objetivos propuestos. Son ajustes en forma progresiva; innovaciones que se implementan en la organización como un todo; deben hacerse con un enfoque sistémico. Las tendencias asociadas a la globalización exigen respuestas 
sólidas y pertinentes a las complejas cadenas de transformaciones y desafíos ante los cuales se encuentran insertas las organizaciones. Al respecto, Hellriegel et. al., (2005) advierten que en algunos casos implica, incluso, un rediseño total de la estructura, una reingeniería; o parcialmente, lo que implica reconfigurar la distribución de la autoridad, mandos medios y controles departamentales y funciones de los empleos; todo ello para ofrecer a la sociedad un servicio de calidad en consecuencia con las necesidades. La organización para implementar los cambios planeados requiere ajustar sus estructuras y sus procedimientos. El cambio no siempre se implementa sin modificar la estructura organizacional, razón por la cual la teoría del desarrollo organizacional ofrece una serie de consideraciones a tomar en cuenta para aplicar transformaciones y conducir la organización hacia el desarrollo.

\section{Desarrollo organizacional, consideraciones}

El desarrollo organizacional es una estrategia debidamente planeada y de proyección futura, sirve para entender, modificar y desarrollar el personal para alcanzar la efectividad, tiene sus orígenes en las ciencias conductuales (Hellriegel et.al., 2005).

Las organizaciones requieren para implementar las acciones conducentes al desarrollo organizacional de una planificación estratégica, entendida esta como la trayectoria impulsada y seleccionada por la dirección de una institución para ejecutar en un plano real la misión de la organización. Es guiar la organización por un camino para llegar al desarrollo organizacional óptimo que le permita alcanzar su misión y visión. Se comprende que para alcanzar la misión en forma eficiente se requiere acompañar la organización de una planificación estratégica; de lo contrario, el desarrollo institucional no logra desarrollarse según lo proyectado.

La planificación estratégica para impulsar el desarrollo organizacional demanda de una revisión en torno a la doctrina organizacional con la cual actúa la institución, donde la visión y misión son los parámetros a considerar en la trayectoria definida. La misión identifica, en forma clara, los valores y el compromiso organizacional en función de la calidad que se desea alcanzar, identifica el compromiso con la razón de ser.

El desarrollo organizacional considera la organización como un todo, no es posible concebir un cambio sin considerar cada una de sus partes, todas tienen una función estratégica, concebida desde la posición que ocupe: es la única forma de alcanzar con mayores posibilidades de efectividad los cambios requeridos (Quiñones, Otarla, Ramos, Aguilera, 2008). Es fundamental para alcanzar los cambios que inspira el desarrollo organizacional considerar distintos componentes:

1. Visión holística de la organización: Cada componente de la organización, desde esta visión del desarrollo organizacional posee un valor fundamental específico; cada parte asume un rol que, unido a otras partes, dan como resultado acciones globales que, en forma individual, no es posible concebir. Cuando una de sus partes no se armoniza, la organización ve limitado su desarrollo efectivo, debe darse una coordinación eficiente entre todas sus partes. Las organizaciones hoy enfrentan en forma constante complejos cambios según las demandas del entorno global, y la única forma de responder con eficacia es que las acciones en el plano organizacional sean 
asumidas desde un liderazgo global que tome en cuenta cada una de sus partes. Para ello se requiere de procesos de gestión competitivos en el plano de la planificación, organización, coordinación y evaluación.

2. Metodología sistémica: Las personas, la estructura y los procesos propios de la organización actúan en forma sistémica, es decir, cada una de sus acciones inciden en todas sus partes, debido a la interconexión en la que actúan. El desarrollo organizacional toma en cuenta esta interacción, comprende que cada una de sus partes afecta los otros componentes y se manifiesta en la organización como un único componente, sin divisiones: es fundamental conocer a cabalidad cada una de sus partes y su funcionamiento. El principio del desarrollo organizacional es articular en forma eficiente cada una de las partes de la organización en función de los objetivos planteados y mantener un constante monitoreo sobre sus comportamientos y sus rendimientos.

3. Designación de agentes de cambio: En todo proceso de desarrollo organizacional existen personas que fungen como agentes de cambio, puede ser más de un individuo. Quienes tienen a cargo la tarea de implementar los cambios necesarios para el desarrollo organizacional les corresponde convencer, estimular y coordinar y gestionar todos los recursos humanos y materiales que conduzcan al objetivo deseado. La selección del agente de cambio es rigurosa, debido a que debe reunir una serie de características personales y profesionales que le trasmitan credibilidad al grupo. Los agentes de cambios pueden ser los directivos o coordinadores de las distintas áreas, pero debe darse la designación. Ningún proceso de cambio se desarrolla sin líder a cargo que realice una adecuada gestión. Hay momentos que las personas que asumen ese papel son externas de la organización, como consultores o consultoras que tienen como ventaja no exponerse ante la organización por las presiones de la jerarquía institucional. Hay organizaciones que, según su capacidad económica, cuentan con un departamento especializado para estos fines o bien poseen profesionales de consultoría externa de manera exclusiva.

4. Identificación real de los problemas: Los procesos de desarrollo organizacional no solo analizan los problemas, sino que su propósito es hacerlo con un abordaje propositivo, identificando las posibles soluciones. Es fundamental la capacidad del equipo encargado para comprender, en forma acertada, el origen y consecuencia del problema: una inadecuada lectura de este hace que cualquier esfuerzo sea en vano.

5. Aprendizaje constructivista: Una de los principios claves del desarrollo organizacional es el aprendizaje práctico. Se parte de que las enseñanzas se interiorizan en la medida que se analice y discuta su propia experiencia, es un enfoque constructivista; construyendo se aprende de una manera más efectiva que comprender situaciones teóricas de manera abstracta. Significa que el desarrollo organizacional efectivo aprende de la propia experiencia.

6. Procesos grupales: El desarrollo grupal más que un proceso individual es colectivo; las organizaciones se conforman de un grupo de individuos, razón por la cual se trabaja hacia la comunidad organizacional más que en el plano individual, a su vez, 
se fortalece la responsabilidad y se construye mayor confianza entre sus integrantes. Son apoyos, en este sentido, todas aquellas actividades que permitan a las personas integrantes de la organización trabajar en forma colaborativa - democrática, para lo que son efectivas actividades de aprendizaje grupal como: análisis de nuevas prácticas, discusiones en grupo, mesas redondas, debates, conflictos intergrupales, análisis de fortalezas, oportunidades, debilidades y amenazas (FODA) de los trabajos en equipo, también procedimientos de coordinación y colaboración. Estas actividades, a su vez, son una oportunidad para mejorar las relaciones interpersonales y los sistemas de comunicación, construir confianza y estimular la responsabilidad entre las personas.

7. Retroalimentación: Desaprovechar oportunidades de retroalimentación no es el sentido del desarrollo organizacional; al contrario, es fundamental ofrecer espacios para la retroalimentación, a fin de que las decisiones tengan mayor fundamento, a su vez son oportunidades de aprendizaje para sus integrantes que permiten asumir acciones autocorrectivas en forma pertinente que conduzcan a la organización, según la trayectoria marcada.

8. Flexibilidad y contingencia: Los procesos de la implementación del desarrollo organizacional, por su misma naturaleza, no son rígidos; se requiere de la flexibilidad, actuando desde el enfoque de la contingencia, es decir, asumiendo los cambios necesarios en el momento oportuno con un enfoque de sistema abierto. Resulta fundamental la capacidad de adaptarse a las distintas situaciones que del mismo proceso emergen. Dentro de estos ajustes siempre se analizan distintas opciones de respuesta, con el objetivo de seleccionar la más adecuada; no se basan en una única manera de plantear los problemas.

9. Trabajo colaborativo: La efectividad del desarrollo organizacional radica en la capacidad de construir estructuras que puedan ser trabajadas en equipo, donde se manifieste la cooperación y la integración como maneras de superar las deficiencias detectadas. Los procedimientos organizacionales son más efectivos cuando los integrantes de la organización se armonizan de manera colectiva para llevar a cabo los diferentes procedimientos que, desde cada posición, sean necesarios. La formación de equipos estimula el aprendizaje y la capacidad de colaborar con compromiso y responsabilidad. Es una estrategia utilizada para implementar los cambios en las organizaciones que les permitan alcanzar su desarrollo.

Con sustento en los planteamientos mencionados, se evidencia que el objetivo fundamental del desarrollo organizacional es la aplicación de procedimientos basados en las ciencias del comportamiento para mejorar la eficacia y eficiencia de las organizaciones; es decir, que el comportamiento del individuo asume relevancia debido a las causas y consecuencias asociadas a su comportamiento ante las demandas que le requiere la organización para implementar los cambios. Para alcanzar este objetivo, hace uso de las competencias de los individuos para poder responder a los cambios del mundo globalizado e incrementar la capacidad de adaptación hacia el medio, de forma que le permita ofrecer respuestas ágiles, sólidas y pertinentes para enfrentar la compleja cadena de transformaciones y desafíos. 
Todas las organizaciones, para asegurar sostenibilidad eficiente, requieren del desarrollo organizacional, con más razón las organizaciones educativas, al tener bajo su responsabilidad la formación constante de las personas mediante el proceso educativo, la producción del conocimiento y la formación de profesionales competentes para responder a las exigencias del escenario global. Ante esto, es importante desarrollar las organizaciones educativas desde un enfoque eficiente, como se plantea por el desarrollo organizacional, y conducir estas organizaciones hacia el logro de los objetivos planteados.

\section{Organizaciones educativas y su desarrollo organizacional}

A inicios de los 80 se dio una connotación especial de parte de los gobiernos, directivas, intelectuales, organismos internacionales como la UNESCO, el Banco Mundial y el Banco Interamericano de Desarrollo, en considerar a la educación como el principal instrumento para la búsqueda del desarrollo social en forma integral, y superar las brechas existentes (Brunner, 2000). La comprensión de este rol dentro de las organizaciones educativas es fundamental para trazar la trayectoria deseada y responder en forma eficiente y eficaz a las transformaciones sociales derivadas del fenómeno de la globalización, la sociedad de la información y el conocimiento. Al apartarse de esta posición epistemológica se corre el riesgo, en las organizaciones, de responder en forma equivocada según sus propósitos.

Uno de los principales retos, y quizás el principal de las organizaciones educativas indistintamente del nivel educativo en el que se encuentren, radica en propiciar las condiciones organizacionales necesarias para que se alcance un nivel óptimo de identidad y cohesión en la organización en busca de la calidad de la educación que se ofrece. Se asume, en igualdad de importancia, en este planteamiento, el desarrollo y la calidad de la educación superior (Alcántara, 2007). Las universidades como organizaciones educativas se enfrentan, en forma constante, a distintos y complejos desafíos por las demandas que el dinámico y desafiante contexto social exige de ellas como organizaciones productoras del conocimiento mediante distintos procesos como la formación de profesionales y la investigación, como parte de su responsabilidad social a la cual se deben.

Los antiguos esquemas de administración y gestión institucional en la educación superior, considera Alcántara (2007) que deben ser remplazados o combinados por nuevas formas de administración tanto en lo estructural como en sus estilos. Estos cambios, producto de las actuales tendencias, sugieren un renovado ambiente organizacional, donde no todos los sistemas podrán responder con la efectividad que requieren, dadas sus características contextuales en materia económica y social.

Históricamente, la educación superior ha asumido como función construir y trasmitir el saber mediante la formación de profesionales para su inserción en el mundo laboral; además, posee un vínculo fundamental con las políticas económicas que dictan el orden social en general (Garbanzo, 2012). De esta forma, aporta los conocimientos necesarios para las transformaciones sociales que el entorno demanda de estas instituciones. Conocimientos que deben ser coherentes en calidad y pertinencia, de acuerdo con las demandas de la internacionacionalización que, debido a los procesos de globalización en que se mueven estas instituciones, les exigen. 
Le corresponde a los distintos niveles educativos y en especial a la educación superior como instrumento potenciador de cambios económicos, sociales y culturales, asumir en forma eficiente sus procesos de gestión; propiciando una educación de mayor inclusión social y, por ende, la aspiración a una sociedad con mayor inclusión, más culta, con un alto nivel de desarrollo, valores éticos y morales. Para lograr esta pertinencia social, es fundamental apoyarse en una gestión participativa, construida en colectividad y con sustentos sólidos con respecto a lo que acontece en el entorno. Estas condiciones son la base para que los procesos de innovación y cambio se produzcan, y para responder a los requerimientos del desarrollo social en forma holística.

Es fundamental que quienes tienen la responsabilidad de conducir estas organizaciones posean conocimiento sólido del acontecer general a nivel mundial, regional y local, y un conocimiento más profundo en el campo específico de la educación superior, para mantener, así, la pertinencia social como institución. Considera Tunnerman (2000) que los esfuerzos por lograr la pertinencia de la educación superior en la sociedad del conocimiento y la información exigen a las universidades replantearse -creativamente- su doctrina organizacional, es decir, sus objetivos, la misión y las funciones. Esto es, reinventarlas en su funcionamiento de manera que estén en articulación con las demandas de la época. Esta condición aplica también para los otros niveles educativos.

Las instituciones de educación superior, además de formar personas competitivas, responsables, solidarias, pro-activas y dispuestas a contribuir al desarrollo de la sociedad globalizada, tiene una doble responsabilidad moral: servir al pueblo y formar profesionales responsables, con ética y altos códigos morales. En este contexto, la pertinencia social debe ser el principal reto desde la gestión de la educación superior que, a criterio de Tunnerman (2000), comprende el compromiso de la educación superior en consecuencia con las necesidades que la sociedad demanda.

La educación superior por naturaleza es compleja, difícil, desigual y cada vez el actual contexto le exige atender una demanda educativa más diversa y dinámica que se refleja en diferentes campos profesionales que emergen del fenómeno de la globalización y la sociedad de la información y el conocimiento. Este contexto hace indispensable que las instituciones de educación superior sean conducidas por líderes capaces de responder a todas las demandas que el entorno nacional y mundial le exige, deben poseer conocimiento necesario sobre el entorno social en todos sus campos -cultural, tecnológico, político, económico y social-. La preparación en materia educativa y una base científica tecnológica es condición para responder, en forma innovadora y crítica, para llevar a cabo los cambios necesarios en forma efectiva y con responsabilidad social a los vertiginosos cambios del contexto al que pertenece.

La globalización impacta sobre los contextos inmediatos en los cuales se desenvuelve la educación, en consecuencia, altera en forma significativa las organizaciones educativas. A criterio de Brunner (2010) se dan transformaciones de fondo en:

- Acceso a la información,

- Acervo de conocimientos

- Mercado laboral

- Disponibilidad de nuevas tecnologías para la educación

- Socialización de los mundos de vida 
Ante los desafíos emergentes, las instituciones educativas en general y con mayor razón la educación superior requieren reorientarse, repensarse en diferentes direcciones mediante la generación de procesos de cambio que permitan el desarrollo de sus propias organizaciones; donde, como lo expone Beck (1999), el conocimiento adquiere un papel transcendental en las economías. Significa que las instituciones educativas, en este campo, para mantenerse posicionadas socialmente deben ser consecuentes con la calidad del conocimiento que ofrecen y, para ello, requieren orientar su gestión hacia el desarrollo organizacional mediante la generación de procesos de cambios necesarios, acordes con las exigencias de los tiempos. La inversión en estos procesos de cambio, ya de por sí mismos, son innovaciones organizacionales a las cuales se les debe apostar.

Estos desafíos los experimentan las organizaciones educativas a lo interno y externo:

Nivel interno: Sus estructuras, las competencias de sus integrantes, procesos curriculares, rendimiento académico, tazas de graduación, procesos de gestión, recursos económicos, procesos de autoevaluación y componentes estructurales, entre otros. Estos elementos por sí mismos son desafiantes y en conjunto producen mayores desafíos, requieren procesos de mejora constante como única forma de responder a las demandas de los tiempos. Las organizaciones que actúan en otras direcciones responden con medios obsoletos que por sí mismos llegan a dejar de tener posicionamiento social; esto en las organizaciones educativas no es una opción, es un constante reto a superar mediante distintos procesos de cambios pertinentes.

Nivel externo: Conformado por las demandas del entorno, modelos económicos y sociales que actúan directamente sobre estas organizaciones producto del fenómeno de la globalización y la sociedad de la información y el conocimiento en el que convergen profundas transformaciones sociales. También, procesos inherentes de las organizaciones de educación superior propios de la actual sociedad, como eficientes procesos de internacionalización y autoevaluación, característicos de las actuales organizaciones en el campo universitario.

Estos niveles provocan procesos de cambio complejos, dinámicos y constantes que las organizaciones educativas deben responder en forma eficiente y asegurar su posicionamiento social. En respuesta a estas transformaciones es necesario considerar una serie de ejes organizacionales en el desarrollo de las instituciones educativas.

\section{Ejes organizacionales para el desarrollo de las instituciones Educativas}

La educación en general y en forma particular la educación superior por naturaleza es compleja, difícil, desigual, por lo que requieren de un enfoque de liderazgo que responda a las complejas necesidades. Administrar, en forma efectiva, en las organizaciones educativas las voluntades humanas y lograr la maximización de las competencias como un todo de sus miembros, para la ejecución de distintos programas y proyectos educativos ya de por sí es un reto. Al respecto, considera Brunner (2010) la necesidad de que en las organizaciones de educación superior se dé un liderazgo de cambio, la ausencia de este produce formas de gobierno débiles. Esta situación 
también aplica para las organizaciones educativas en general, no es una condición exclusiva de la educación superior. Estas formas de gobierno limitan la gestión de las organizaciones educativas para responder, en forma acertada, ante las demandas emergentes sociales.

El liderazgo de la educación requiere habilidades efectivas de comunicación, persuasión, relaciones humanas y negociación, que representan los cimientos para que surjan condiciones organizacionales mínimas que permitan la operacionalización efectiva de sus propios objetivos. Estas condiciones mínimas se traducen en satisfacción laboral, clima organizacional adecuado, altos niveles de motivación y, por ende, compromiso y responsabilidad con altos códigos éticos y morales. En este contexto, seguidamente se desarrollan distintos ejes fundamentales a considerar para el desarrollo de las instituciones educativas:

1. Liderazago innovador: Es un liderazgo que responde con propuestas transformadoras, enfocadas hacia el desarrollo según las exigencias de la modernidad, y oportunas con viabilidad de implementación desde una visión de futuro holística. Además de innovador es independiente y comprometido, actúa con responsabilidad y transparencia. Un liderazgo con visión competitiva ante la complejidad, con un espíritu crítico y capacidad de leer las demandas contextuales en forma acertada. Esta condición le permite asumir un liderazgo estratégico, político, pedagógico. Tiene capacidad para manejar la incertidumbre, los conflictos, la complejidad y tomar acertadas decisiones en estos ambientes desafiantes, así como asumir las consecuencias de las decisiones. Trabaja en forma planificada, lo que le permite hacer un correcto manejo de la incertidumbre, la complejidad y los conflictos, para construir un futuro acertado sobre el presente. Las decisiones son debidamente planificadas, lo que le permite gestionar, direccionar y controlar lo que sea necesario desde los propios procesos internos de gestión, con perspectiva de futuro y sistémica, sin descuidar las dimensiones de corto y mediano plazo. Se asume un liderazgo con capacidad política, destrezas para unir voluntades, que incrementa la capacidad de gestión. Es una gestión con habilidad para la internalización, formación académica satisfactoria, habilidades humanas y altos valores éticos y morales.

2. Manejo de transiciones: Las organizaciones, al igual que toda unidad sistémica, experimenta la incorporación de nuevos miembros en sus estructuras, nuevos líderes. Son transiciones donde la clave no es desplazar en su totalidad una cultura organizacional; por el contrario, el reconocimiento de las experiencias y contribuciones viene a ser un factor estratégico en el éxito del desarrollo organizacional. El liderazgo debe ser capaz de reconocer las riquezas de la organización, los aportes de sus antecesores y sobre este presente construir el futuro deseado. Es obligación del liderazgo organizacional eficiente dejar huellas de crecimiento, de desarrollo en la gestión, pues son las bases sólidas de las nuevas generaciones.

3. Conocimiento del capital humano: El éxito o el fracaso en el desarrollo organizacional, por lo general, está determinado por la habilidad del liderazgo para saber tratar con las distintas personas que conforman la organización. Lograr la interacción entre sus características individuales y grupales es la habilidad del liderazgo para las relaciones interpersonales (Chivanenato, 2009). Esta condición es una base sólida para implementar los cambios requeridos para el desarrollo institucional de las organizaciones educativas. 
4. Construir y reconstruir el saber: Son procesos de cambios generados en busca del desarrollo organizacional y la capacidad de la organización de invertir generando espacios para construir y reconstruir el saber en forma continua (Beck, 1999). La organización educativa comprende que sus propuestas curriculares tienen una validez definida, las demandas sociales son cambiantes, razón por la cual es necesario gestionar procesos de actualización y formación profesional de su personal para asegurarse la actualización permanente de sus propuestas curriculares en consecuencia con las demandas, para lo se requiere de equipos de alto nivel que, además de ofrecer respuestas curriculares novedosas, sean capaces de hacer lecturas acertadas de las demandas a las cuales se deben las organizaciones educativas. Se requieren propuestas con carácter transnacional, interdisciplinarios, currículos globales multidimensionales.

5. Redes de investigación: Las tendencias mundiales asociadas al fenómeno de la globalización y de la sociedad de la información y el conocimiento han traído consigo, para las organizaciones educativas, desafiantes transformaciones en sus maneras de producir y trasmitir conocimiento. El conocimiento ha dejado de tener una perspectiva local para asumir un carácter global, lo que ha generado nuevos procedimientos de construcción desde lo interdisciplinario y global mediante la tecnología emergente, como lo vienen a ser los ya estructurados sistemas de redes de investigación a nivel nacional y mundial. La incorporación de las tecnologías en las organizaciones educativas exigieron una redirección de la gestión de estas instituciones, asumiendo cambios en sus estructuras para implementar procesos de innovación en la producción y transmisión del conocimiento en procura de ofrecer mayores oportunidades al desarrollo social. Las instituciones educativas en su mayoría han incorporado departamentos, coordinaciones, trabajos en equipos que le den sostenibilidad a las redes de investigación según las necesidades del nuevo escenario.

6. Redes de cooperación: A finales de la década de los 90 Castells (1997) advertía que la organización de la educación en el futuro, en consecuencia con la configuración social, sería una sociedad en redes, visión futurista acertada. Las transformaciones sociales han impactado en las estructuras organizacionales para producir cambios y desarrollar innovaciones en sus modos de trabajo, lo que supone que las organizaciones educativas han desplazado sus formas de trabajo tradicionales centralizadas, burocráticas, rígidas pasando a una organización en redes, flexible, de cooperación, dinámica, estructuras abiertas, límites no determinados en forma permanente, jerarquías articuladas, descentralizadas, variadas conexiones, ágiles sistemas de comunicación y eficientes, de acuerdo con las necesidades del escenario globalizado.

De esta forma, uno de los cambios implementados en el desarrollo organizacional de las instituciones educativas en respuesta a la compleja cadena de transformaciones sociales han sido innovaciones en las formas de trabajar, dejando a un lado el trabajo más localizado para darle lugar a formas de trabajar basadas en la cooperación mediante redes; estas no son exclusivamente a nivel institucional, sino que se articulan en forma interinstitucional tanto a escala nacional como internacional. Estas redes de cooperación permiten compartir los conocimientos y apoyar procesos haciendo uso de variados saberes; indistintamente de donde se produzca el intercambio académico, se ha 
incrementado de manera acelerada con plataformas tecnológicas que también posibilitan estas estructuras de cooperación. Este incremento no parece mermar, al contrario, el futuro de las instituciones educativas se direcciona hacia estas formas de trabajar y, en algunos casos, los espacios físicos dejarán de ocupar el lugar que han tenido.

7. Políticas de internacionalización: Traspasar fronteras, mediante la articulación internacional, modificando e implementado cambios para innovar en sus estrategias. En el caso de las universidades, no es la universidad que piensa en sí misma, es desde sí misma hacia lo global, hacia lo internacional, entran redes de gestión, de investigación, de personal académico, movilidad estudiantil y de proyección social. Este componente de internacionalización es cada día más articulado en los otros niveles educativos, como por ejemplo en los programas de bachillerato internacional en la educación secundaria, auge que ha venido a fomentar importantes innovaciones, producto de los cambios que las organizaciones educativas han tenido que asumir para responderle a las necesidades del complejo entorno.

8. Calidad y pertinencia educativa: La calidad y la pertinencia, más que una aspiración, es una condición. Se requieren renovados planes de desarrollo institucionales donde se consideren los distintos campos del saber que ofrece la institución, lo que implica una apertura flexible a renovar sus estructuras en busca de la pertinencia institucional; estas flexibilidades vienen a representar innovaciones generadas de los cambios en los que se han visto enfrentadas las instituciones educativas. Son importantes políticas de producción del conocimiento con alto valor ético y social, lo mismo que políticas de acceso y equidad educativa.

9. Ofertas innovadoras: Las organizaciones educativas, para asegurar su sostenibilidad, han tenido y tendrán que implementar cambios que les permitan innovar mediante la producción de ofertas educativas acordes con las demandas contextuales. Conforme avanzan las tendencias mundiales asociadas a la globalización y a la sociedad de la información y el conocimiento vienen consigo complejas y variadas transformaciones que requieren de las instituciones de educación superior renovadas ofertas educativas. Estas ofertas demandan una constante actualización para no perder viabilidad social. Esta actualización constante por sí misma es considerada una innovación organizacional que cada institución produce en respuesta a los cambios que debe ofrecer para garantizar su legitimidad y posicionamiento social; de lo contrario, puede llegar a desaparecer.

10. Capacidad de comprensión de sí misma en su totalidad y partes: Senge (1990, citado por Acosta, 2011) le atribuye a las organizaciones capaces de desarrollarse la capacidad de comprender su totalidad desde un enfoque sistémico, de manera que, además de su globalidad, se comprenda cada una de sus partes y su funcionamiento en forma individual e integral.

En consecuencia, aquellas organizaciones que aspiran el desarrollo organizacional requieren tomar en cuenta estos ejes, los cuales vienen a representar un camino para generar condiciones para una organización inteligente, capaz de responder a las demandas de su entorno en forma eficiente, basada en el aprendizaje organizacional tal y como se expone seguidamente. 


\section{Desarrollo organizacional y aprendizaje organizacional: Condiciones para una organización inteligente}

La capacidad organizacional de aprendizaje por sí misma es una innovación que orienta el desarrollo organizacional, este influye en forma positiva en el desarrollo de innovaciones y en una gestión competitiva organizacional. Se considerada el aprendizaje como una condición eminente para el cambio sustentado en el nivel de conocimiento de cada organización, que incluye tanto componentes cognitivos y de comportamiento asociados al conocimiento.

Hellriegel et al. (2005, p. 345) definen la innovación como "el proceso de crear y poner en práctica una idea nueva". La innovación siempre conlleva la ruptura o la redefinición de algún procedimiento o el surgimiento de un pensamiento emergente, que puesto en práctica aporta en nuevos esquemas organizacionales en busca de los objetivos institucionales; es hacer algo nuevo, diferente. Las ideas que sustenta la innovación pueden surgir a lo interno de la organización como a lo externo. Los ambientes organizacionales dinámicos promueven con mayor facilidad el cambio y la innovación. Las innovaciones pueden producirse en lo técnico, en procesos y en los procedimientos propios de la administración.

La innovación conlleva cambios profundos y respuestas transformadoras a las demandas contextuales, que a su vez se traducen en desarrollo de competencias laborales del personal miembro de la organización, y generar un mayor crecimiento de la organización y capacidad de adaptativa ejemplar en forma permanente, entendiendo la organización desde un enfoque sistémico.

No existe una única metodología aplicable a todas las organizaciones, y significa un cambio drástico en cómo pensar y hacer las distintas funciones que dentro de la organización se esperan de cada funcionario o funcionaria según la posición que ocupen. El producto es un nuevo aprendizaje con mayor eficiencia o un aprendizaje estructurado hacia el desarrollo. Este aprendizaje se produce en distintos niveles: en los individuos, en las gerencias, equipos de trabajo y en redes organizacionales tanto internas como externas.

El aprendizaje organizacional es un proceso sistémico, que posee una estructura de conocimientos que evolucionan, se desarrollan en forma permanente respondiendo a las transformaciones del contexto que se producen en variadas formas según sea cada caso. De manera que la innovación se comprende como un proceso que articula la acumulación y combinación de nuevos conocimientos (Acosta, 2011).

Una organización que aprende cuenta con los recursos internos necesarios para impulsar las transformaciones que requiere, así como también posee capacidad de impulsar una cultura de mejora permanente y optimar el desempeño de cada individuo que la conforma. En las organizaciones de aprendizaje, la innovación y el cambio se ven como procesos propios de la cultura interna. Existen características de las organizaciones que aprenden, dignas de considerar en el campo educativo:

- El liderazgo es compartido.

- La innovación es parte de la cultura. 
- La atención a la población meta es prioritaria.

- Los miembros de la organización son parte de la identificación y solución de problemas.

- La resistencia laboral es enfocada en forma acertada.

- Respecto a los medios de comunicación, tanto la gerencia como el resto de los niveles conocen y comparten los alcances, objetivos y metas.

Además de estas características, las organizaciones educativas que aprenden en sí mismas requieren, desde su gestión, considerar distintos elementos que, articulados, propician la eficiencia organizacional con base en el aprendizaje mutuo, para considerarse organizaciones inteligentes.

\section{Propuesta}

A partir de lo anterior, se proponen los siguientes elementos que serán fundamentales a tomar en cuenta por la administración de la educación en sus procesos de gestión, que permitan a las organizaciones educativas actuar en eficiencia como organización inteligente para lograr los objetivos planteados.

Competitividad: el aprendizaje permite que la organización sea eficiente, obtenga los resultados por los cuales nació, según sus objetivos, con altos niveles de competitividad de acuerdo con las demandas del contexto a las cuales se debe.

Ambiente permanente de cambio: La adquisición del aprendizaje es la base para alcanzar el éxito y generar procesos de cambio acertados, se comparte esta posición cognitiva.

Visión holística, sistémica: Se comprende que toda acción repercute en la organización como un todo y en cada una de sus partes, estas consecuencias son tanto en el presente como el futuro y son acompañadas de una gestión que comparte y ejecuta esta visión.

Visión a futuro: Se trabaja en el presente en función del futuro, estos principios son comprendidos por la gestión y trasmitidos e interiorizados por todos los integrantes de la organización.

Trabajo en equipo: Fundamental para alcanzar los propósitos organizacionales que por sí mismos no es posible alcanzar en forma individual. No hay organización que con una ejecución unilateral y un trabajo individual de parte de sus integrantes alcance el cumplimiento de sus objetivos en forma adecuada.

Relaciones humanas satisfactorias: El componente humano es una característica fundamental, que coadyuva a establecer relaciones con ética, creatividad, innovación, respeto. Todos estos elementos desde un enfoque sistémico proporcionan estructuras adecuadas para el desempeño de la organización y deben ser generadas e impulsadas por la gestión.

Alto nivel de compromiso y responsabilidad: La capacidad organizacional de contar con altos niveles de compromiso y responsabilidad de parte de sus integrantes en función del cumplimiento de la misión es la condición deseada para cualquier organización, que a su vez es un reto de la gestión. Estas condiciones se viven y se modelan desde la gestión y se interiorizan como una cultura laboral. 
Gestión del conocimiento: El conocimiento individual y grupal de una organización es un capital intelectual que lidera el posicionamiento de la organización y, así como otros recursos, este requiere de una eficiente gestión. El conocimiento por sí mismo no se traduce en acciones, requiere de una gestión competitiva que impulse las condiciones necesarias para que el conocimiento que posee la organización se transforme en servicios de alta calidad.

\section{Consideraciones finales}

El liderazgo compartido mediante una eficiente gestión es clave en las organizaciones que aprenden; la responsabilidad, la dirección de los procesos y el cumplimiento de los objetivos se comparten en la organización en forma general y se trabaja con un enfoque sistémico; la totalidad de sus miembros se siente parte de la institución. Se experimenta un ambiente de confianza y espíritu colaborativo. La innovación es una característica de la cultura, es parte de ella: se experimenta libertad para proponer y ser parte. Estas organizaciones tienen rápida capacidad para adaptarse a sus prácticas y satisfacer las necesidades de la población a la cual se dirigen.

Las organizaciones inteligentes invierten en el aprendizaje y comprenden que las organizaciones que sean incapaces de aprender desde un enfoque sistémico y permanente tienen pocas posibilidades de sostenerse en el contexto ante el cual fueron creadas. Una organización inteligente comprende que invertir en el aprendizaje no es una opción, es una responsabilidad que se debe asumir con los más altos principios y valores éticos y morales. Así, una organización de aprendizaje es capaz de crear, adquirir, trasmitir y reformar actitudes que promueven el desarrollo individual dentro de una organización que se enfrenta a constantes transformaciones (Chiavenato, 2009). Cuando el aprendizaje individual aumenta, también aumenta el capital intelectual de la organización y le corresponde a la gestión transformar este conocimiento en acción.

Cuanta mayor capacidad de aprendizaje tiene la organización como sistema, son más las probabilidades de alcanzar procesos de innovación y la competitividad en forma sostenible: no solo es alcanzar los procesos de desarrollo mediante la innovación, es la capacidad del sistema de mantenerse en esta condición, lo cual es la clave para cualquier desarrollo organizacional. De esta forma, las organizaciones son capaces de producir conocimiento, y este a su vez es dinámico, implica un proceso de conocer y, por ende, capacidad para actuar y transformar; de lo contrario, el conocimiento asume un carácter latente, se trasmitió pero no hubo dinamismo ni capacidad de aplicar lo adquirido, es decir, no se dio aprendizaje porque este es el que permite la acción.

El aprendizaje organizacional a su vez es un proceso de adaptación del entorno, donde el conocimiento de la trayectoria organizacional producto de la relación con el entorno permite la adaptación y sostenibilidad ante la desafiante sociedad.

El aprendizaje individual se transforma en aprendizaje organizacional cuando las organizaciones, mediante sus procesos de gestión internos, logran esta articulación; estos resultados las hacen merecedoras de considerarse organizaciones inteligentes. Se producen conocimientos producto de la trayectoria organizacional en la resolución de los distintos problemas, transformación de rutinas, procedimientos, enfoques y normativas que les permiten responder en forma acertada al entorno (Acosta, 2011). 
El aprendizaje organizacional tiene una importante asociación con el desarrollo de las innovaciones. Este aprendizaje que se visualiza en dos niveles, en el plano individual y colectivo, va a generar las bases sobre las cuales afloran las innovaciones, y estas, a su vez, son la base para el desarrollo organizacional. La organización que aprende es aquella que desarrolla una importante capacidad de aprender en forma continua y desarrolla una capacidad de adaptación y cambio continuo (Chiavenato, 2009). Estos procesos se dan en forma consciente o inconsciente; se requiere de una gestión que comprenda en general este aprendizaje y que haga comprender a la organización la capacidad organizacional, como colectivo, y conduzca a sus integrantes para su aplicación, según los objetivos organizacionales.

En las organizaciones inteligentes, de aprendizaje, se comprende que todas las personas sin distinción de ocupación tienen conocimiento y poseen la capacidad como colectivo de transformarlo en acciones. Ese proceso de convertir el conocimiento en acción no todas la organizaciones lo logran, y la diferencia radica en la comprensión común de la misión y visión, así como una gestión que conduzca a este nivel de aprendizaje en acción. Importante tomar en cuenta que son los individuos quienes desarrollan y transforman las organizaciones, mediante ellos el conocimiento asume carácter de acción.

La habilidad de la gerencia, la dirección de trabajar con todo el conocimiento que poseen sus integrantes es la clave; el personal directivo debe, como responsabilidad para el cumplimiento de los objetivos, transformar el conocimiento que posee cada quien y luego articular la suma total del conocimiento de la totalidad de sus miembros y obtener, como resultado, la acciones necesarias mediante procesos de innovación para la sostenibilidad de una organización inteligente, de aprendizaje que responda en forma eficiente a las demandas contextuales, según sus objetivos desde un enfoque sistémico.

En síntesis, la capacidad de aprendizaje de una organización educativa es la condición necesaria e indispensable para generar cambios mediante procesos de innovación. La implementación efectiva de estos cambios requiere de distintos procesos a considerar como evaluar el medio ambiente, determinar la brecha del desempeño, diagnosticar problemas organizacionales, articular y comunicar una visión para el futuro, desarrollar y poner en práctica el plan acción, anticipar las resistencia y tomar acciones para reducirla. Todos estos procesos son indispensables para responder en forma efectiva a los desafíos que la sociedad le impone a las organizaciones, indistintamente de su campo de acción.

Una organización que está en equilibrio interno es una organización en condiciones de responder al entorno en condiciones satisfactorias. Se comprende que el liderazgo requiere de una articulación entre el líder, seguidores y contexto tal y como lo expone Hesselbein, Goldsmith, Benckard (2006). Esta capacidad de respuesta hacia el entorno lo determina la gestión, la cual debe ser basada en un liderazgo transformador, fundamentado en una cultura de competitividad en forma permanente, es un paradigma de gerencia moderna.

Las organizaciones inteligentes invierten en el aprendizaje y asumen el compromiso de aprender en forma sistémica y permanente; principios que las conducen a alcanzar sostenibilidad y legitimidad social. La inversión en el aprendizaje no es una opción, se considera una responsabilidad que debe acompañarse de valores éticos y morales. Cuando el aprendizaje individual incrementa, incide en el capital intelectual de la organización y es 
tarea de la gestión transformar el conocimiento en acción, lo que le dará mayores posibilidades de ejecutar procesos de cambio y de innovación en forma sostenible.

La gestión de las organizaciones educativas de aprendizaje e inteligentes direcciona sus esfuerzos para responder en forma eficiente y eficaz ante las complejas transformaciones que la sociedad espera de ellas, y se reconocen por respuestas acertadas en diferentes campos. Las organizaciones educativas inteligentes son capaces de llevar a cabo sus procesos de cambio, según propuestas de planeación institucional, estas no se quedan en el papel; además de tener la capacidad de determinar sus programas, proyectos, estrategias y acciones, también saben cómo transformarlas en acciones, superan el nivel documental de la planeación institucional. Muchas organizaciones tienen la capacidad de generar ideas, planteamientos, saber qué hacer; pero son pocas las que tienen la capacidad de transformar sus propuestas de planeación institucional en acción. El gran motor que conduce la operacionalización de estas propuestas tiene la base en un liderazgo transformacional, el cual difiere de la fama y el poder, condiciones que no son, por sí mismas, efectivas. Un acertado liderazgo sabe que requiere de la interacción tanto de líderes, seguidores y contexto para poder transformar la misión organizacional en acciones y estas en una formación educativa acorde con el contexto demandante.

\section{Referencias}

Alcántara, A. (2007). Tenencias mundiales en la educación superior: El papel de los organismos multilaterales. Revista Inter Ação 31 (1): 11-33. doi: http://dx.doi.org/10.5216/ ia.v31i1.1490

Acosta, P. (2011). El aprendizaje organizacional como proceso de innovación en nuevas empresas de base tecnológica, un estudio de casos. Ideas, CONCYTEG 6 (74) 959-976.

Beck, U. (1999). ¿Qué es la globalización? Falacias del globalismo, respuestas a la globalización. Barcelona: Paidos Ibérica.

Brunner, J. (2000). Globalización y el futuro de la educación. Seminario sobre prospectiva de la educación en la región de América Latina y el Caribe. Santiago: UNESCO. Recuperado de http://mt.educarchile.cl/archives/Futuro_EDU\%25UNESCO-2000.pdf

Brunner, J. (2010). Educación superior en América Latina. Santiago: Centro de Políticas Comparadas en Educación.

Castells, M. (1997). The Information Age: The Rise of the Network Society [La era de la información: El auge de la sociedad red]. Oxford: Blackwell Publishers.

Chiavenato, I. (2009). Comportamiento organizacional. (2 Ed.), México: Mc-Graw Hill. 
Garbanzo, G. (enero-abril, 2012). Educación superior pública en América Latina: Características y desafíos. Revista Gestao Universitaria na America Latina - GUAL- 5(1): 216-227. doi: http://dx.doi.org/10.5007/1983-4535.2012v5n1p216.

Hellriegel, D., Jackson, S. y Solcum, J. (2005). Administración. Un enfoque basado en competencias (10 Ed.). México: McGraw-Hill de México.

Hesselbein, F., Goldsmith, M., y Benckhard, R. (2006). El líder del futuro. Barcelona: Ediciones Deusto.

Quiñones, V., Otarla, S., Ramos, F. y Aguilera, N. (2008). Desarrollo organizacional. Blogspot, Grupo15. Recuperado de https://www.bing.com/search?q=Desarrollo+Organizacional.+Bl ogspot\%2C+Grupo15.\&PC=U316\&FORM=CHROMN

Tünnerman, C. (2000). Pertinencia social y principios básicos para orientar el diseño de políticas de educación superior. Educación Superior Sociedad 11(1), 181-196. Recuperado de http://ess.iesalc.unesco.org.ve/index.php/ess/article/viewArticle/364 